\title{
Analysis on Topological Properties of Dalian Hazardous Materials Road Transportation Network
}

\author{
Pengyun Chong, ${ }^{1}$ Bin Shuai, ${ }^{1}$ Shaowei Deng, ${ }^{1}$ Jianting Yang, ${ }^{2}$ and Hui Yin ${ }^{3}$ \\ ${ }^{1}$ School of Transportation and Logistics, Southwest Jiaotong University, Chengdu 610031, China \\ ${ }^{2}$ Faculty of Geosciences and Environmental Engineering, Southwest Jiaotong University, Chengdu 610031, China \\ ${ }^{3}$ Kunming Hydroelectric Investigation Design and Research Institute, China Hydropower Engineering Consulting Group Corporation, \\ Kunming 650051, China
}

Correspondence should be addressed to Bin Shuai; bsh67@126.com

Received 19 July 2014; Accepted 10 November 2014

Academic Editor: Hong Chen

Copyright (C) 2015 Pengyun Chong et al. This is an open access article distributed under the Creative Commons Attribution License, which permits unrestricted use, distribution, and reproduction in any medium, provided the original work is properly cited.

To analyze the topological properties of hazardous materials road transportation network (HMRTN), this paper proposed two different ways to construct the cyberspace of HMRTN and constructed their complex network models, respectively. One was the physical network model of HMRTN based on the primal approach and the other was the service network model of HMRTN based on neighboring nodes. The two complex network models were built by using the case of Dalian HMRTN. The physical network model contained 154 nodes and 238 edges, and the statistical analysis results showed that (1) the cumulative node degree of physical network was subjected to exponential distribution, showing the network properties of random network and that (2) the HMRTN had small characteristic path length and large network clustering coefficient, which was a typical small-world network. The service network model contained 569 nodes and 1318 edges, and the statistical analysis results showed that (1) the cumulative node degree of service network was subjected to power-law distribution, showing the network properties of scale-free network and that (2) the relationship between nodes strength and their descending order ordinal and the relationship between nodes strength and cumulative nodes strength were both subjected to power-law distribution, also showing the network properties of scale-free network.

\section{Introduction}

Complexity science is the kind of science of the 21st century. Complex network has developed rapidly in the past few years as an important part of the complexity science [1]. Its main idea is to convert the various elements within the research system into nodes of the network, and the relationship between the elements into the edges to form a network in order to describe the relationship among the various elements within the research system. Exploring the essential properties of the real system through the studies of the topological properties of the network structure is an effective method to research complexity science.

Mathematical theory basis of complex network theory is graph theory. Euler's study on "seven-bridge problem" of Gurney Myers in 1736 was the beginning of the studies into complex network theory. Since then people abstracted a number of complex systems into networks to describe and research from the perspective of the network topology, such as the Internet network [2], the food chain network [3], neural networks [4], and the citation network [5]. Milgram first proposed small-world phenomenon of network in 1967 in the analysis of network structure [6]. Based on this, Watts and Strogatz proposed WS small-world network in the study of small-world phenomenon generation process mechanisms and presented the network generation algorithm for the first time in 1998 [7]. Barabási and Albert found the scale-free properties of real-world networks in 1999, which called BA model, and presented the network generation algorithm [8]. The research results of Watts and Barabási et al. subverted people's awareness of the traditional network, causing many scholars in the field of physics, economics, and computer communications to focus on complex network [9-11]. 
The normal operation of traffic and transportation system is an important prerequisite for the steady and rapid development of the national economic, and the complex network theory has attracted wide attention of scholars in transportation field. In current studies, scholars research the topological properties of the transportation network mainly by means of graph theory and network topology theory and so on. Transportation network has growth and irregularity; therefore, these theories can describe networks of simple structure well, but not competent for complex transportation network. The research results of application of complex network theory to the transportation network topological properties are mainly concentrated on the railway network [12-14], airport network [15-20], and urban traffic network $[10,21]$.

In the studies on topological properties of railway network, Sen et al. [12] studied the Indian railway network in the $\mathrm{P}$ space, which showed that it had a shorter average path length and large clustering coefficient, presenting smallworld network properties. Li and Cai [13] researched China railway passenger transportation network, and the results showed that the physical railway network had the tree structure and the traffic flow network was a typical small-world network. Kurant and Thiran [14] made an empirical study on the three kinds of space forms P, R, and L of European and Swiss railway networks and analyzed the similarities and differences among the railway network topological properties of different space forms.

In the studies on topological properties of airport network, Guimerà et al. $[15,16]$ researched global airport networks, and results showed that the airline network was a small-world network, with average shortest path length of 4.4 and the network average clustering coefficient of 0.62 . Chi et al. [17] constructed a directed and weighted airport network of USA, with similar research results to Guimerà et al, that is, American airport network also presenting smallworld properties, with average shortest path length of 2.4 and the network average clustering coefficient of 0.618 . Other scholars have conducted similar studies, such as Bagler [18] on India airport network, Guida and Maria [19] on Italy airport network, and $\mathrm{Li}$ and Cai [20] on Chinese airport network. Their results showed that the airport network was small-world network, and the network average clustering coefficient was similar. The only difference was that the average shortest path length of global airport network was greater than that of each national airport networks.

In the studies on topological properties of urban traffic network, Latora and Marchiori [10] researched the complex network properties of the Boston subway network by constructing an undirected and weighted subway network, and results showed that the Boston subway network presented small-world properties. Jiang and Claramunt [21] researched urban road network, and results showed that it presented small-world network properties.

In general, the studies on complex network are mainly focused on the following areas: (1) to simulate the geometrical statistics (such as the number of nodes, the number of edges, the network average node degree) of real world by using generation algorithm of random network, scale-free network, and small-world network [7, 8]; (2) to research dynamic problems of network topology structure [22]; (3) to observe the change of its properties after the implementation of different operation on the network, that is, to research network survivability at different attack strategies [23]. Studies of applying complex network theory to researching the transportation network can be divided roughly into two categories: (1) to research the topological properties of the transportation network, which mostly concentrated on the railway network [12-14], airport network [15-20], urban rail transit network [10], and urban road network [21]; (2) to study how to improve the reliability and survivability of the network under a deliberate or random attack, optimizing network structure when the topological properties of the network are known [24].

Compared with the common freight transportation, hazardous materials transportation objects are flammable, explosive, corrosive, and radioactive such as gasoline, explosive, strong acid, and peroxide. So once an accident happens during the transport process, it will cause serious damage to the environment, the surrounding residents, and the normal operation of economic activity. At present, China railway, airport, and shipping are not perfect in hazardous materials transportation infrastructure and management mechanism, so road transportation is the main way of the hazardous materials transportation. According to statistics, road transports cover about $80 \%$ of our hazardous materials [25]. In 2009, China's annual hazardous materials transport volume is more than 4 million tons, and in the first half of 2013, the total increase in main chemicals is about $6.3 \%$, and more than $95 \%$ of the hazardous materials relate to offsite transportation problem [25], so hazardous materials transportation in our country presents properties of having a large volume, a high growth rate, a single mode of transportation, a low safety management level, and so on. Due to the increasing transport volume of hazardous materials, both road mileage and density of road network of hazardous materials transportation network increase significantly, and also the integration and the complexity of the network are more obvious.

Compared to other transportation networks, hazardous materials road transportation network (HMRTN) is a special kind of transit network, which mainly reflected on the following points. (1) Different network planning concept: for common freight transport, the general intention of road network planning is mainly reducing running costs or shortening the distance between two places. Because of the particularity of hazardous materials, transportation risk must be minimized. So the basic principle of HMRTN planning is to minimize risk, which also leads to differences between that and the other road transportation network. (2) Different network system composing elements: edges and nodes of HMRTN should be far away from area of high network density, such as population, schools, water, bridges, and government agencies, which leads to its network structure different from other transportation network. (3) Different network scale: edges and nodes of HMRTN are composed of the city's main roads and intersections, which is a part of urban road network but different from it. Considering the several factors above, HMRTN must present different topological properties from 
the other transportation network. When hazardous materials transportation vehicles encounter random attacks (such as traffic accidents) or deliberate attacks (like terrorist attacks) in the transport process, transportation network function will be impaired. In particular, when the accident locates at the network node or edge which is the most "fragile," it may paralyze the network. Therefore, applying complex network theory to researching topological properties of HMRTN helps to improve the understanding of the properties of network and reduce the damage of the unknown risks to network function.

Based on the above analysis, this paper researches the topological properties of HMRTN. On the basis of constructing hazardous materials road transportation physical network and service network structure models, and analyzing the interaction between them, this paper constructs the physical network model of HMRTN based on the primal approach and the service network model of HMRTN based on the neighboring nodes by applying complex network theory and puts forward the research process of network topological properties. The method is applied to the Dalian HMRTN. This paper researches the complex network properties of HMRTN through the statistical average shortest path length, the network diameter, average network clustering coefficient, node degree, strength, the strength parameters, and so on and provides scientific basis for the planning and optimization of HMRTN.

\section{Methodology of Researching Topological Properties of HMRTN}

In order to get the network topological properties, the following questions need to be focused on for the research method of hazardous materials road transportation network properties. (1) What is HMRTN? (2) What factors could be used to judge the topological properties of a network? (3) How to judge the type of a complex network? (4) How to construct a HMRTN based on complex network theory? (5) What is the research process of network topological properties? In view of the above questions, the main methods of this paper are explained through the following aspects.

2.1. The Structure Model of HMRTN. HMRTN system is composed of nodes of different nature, the road network of connection among the nodes, and the OD pairs' information with distribution tasks. Therefore, HMRTN includes physical network and service network, and it has the double attributes. Based on this, this paper constructs its network models, respectively.

(1) Physical Network Space. The network $G=(V, E)$ in urban road network represents hazardous materials road transportation physical network. $V$ is a node set of network $G$ that represents the places of actual meaning like intersections, and so forth. If $|V|=m_{a}$, say network $G$ has $m_{a}$ nodes, $V=\left\{v_{1}, v_{2}, v_{3}, \ldots, v_{m_{a}}\right\} ; E$ is the edge set of network $G$, which represents the connection path between two nodes, including expressway, national highway, and the urban road.
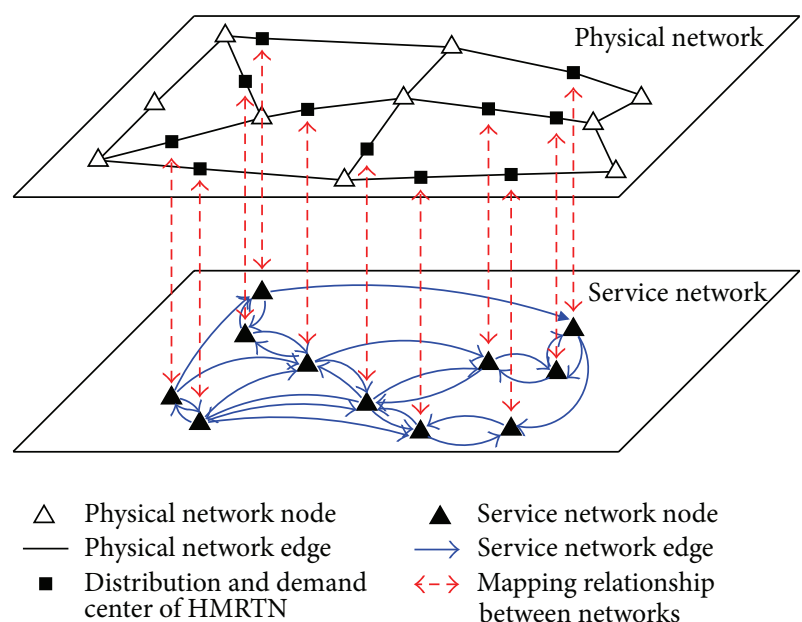

FIGURE 1: The spatial view of physical network/service network of HMRTN.

If $|E|=n_{a}$, representing $n_{a}$ edges in network $G, E=$ $\left\{e_{1}, e_{2}, e_{3}, \ldots, e_{n_{a}}\right\} ; W$ are the edge weights of network $G$, which represents a set of path lengths between any two nodes; that is, $W=\left\{w_{1}, w_{2}, w_{3}, \ldots, w_{n_{a}}\right\}$. Therefore, the physical network constructed in this paper is an undirected, weighted, and connected network.

(2) Service Network Space. In hazardous materials road transportation physical network $G=(V, E)$, where $s_{i, j}$ is the minimum risk path between any two nodes $v_{i}$ and $v_{j}$, hazardous materials road transportation vehicles complete the distribution task along the minimum risk path. In the distribution process, transportation vehicles passing distribution center, demand center, and distribution route compose another huge service network $G^{\prime}=\left(V^{\prime}, E^{\prime}\right)$, where $V^{\prime}$ is the places of actual meaning like hazardous materials distribution center, demand center, and so forth. If $\left|V^{\prime}\right|=m_{b}$, which represents $m_{b}$ nodes in network $G^{\prime}, V^{\prime}=\left\{v_{1}, v_{2}, v_{3}, \ldots, v_{m_{b}}\right\}$. $E^{\prime}$ is the network edge set formed by distribution route passing two neighboring nodes. Because the distribution is divided into upstream and downstream, the edges in network $G^{\prime}$ are directed edges. If $\left|\vec{E}^{\prime}\right|=n_{b}$, which represents $n_{b}$ edges in network $G^{\prime}, \vec{E}^{\prime}=\left\{e_{1,2}, e_{2,1}, e_{3,4}, \ldots, e_{i, j}\right\}, i, j \in m_{b}, i \neq j$. Therefore, the service network in this paper is a directed, weighted, and connected network.

HMRTN model and spatial interaction are shown in Figure 1.

2.2. Methodology of Establishing the Complex Network Model of HMRTN. Based on the above analysis, the physical network and service network of hazardous materials road transportation complex network models are constructed as follows.

2.2.1. The Physical Network Model of HMRTN Based on Primal Approach. To ensure abstract network reflecting the spatial relationship between the network topological properties and road intersection utmost well, in this paper, a hazardous 
materials road transportation physical network model based on the primal approach is constructed. The primal approach refers to the road path abstracted to network edges and the intersections to network nodes [26]. And the following basic definitions and assumptions are made to the network topology.

(1) Do not consider the properties and ranking differences of HMRTN edges and nodes.

(2) Do not consider the direction of the road, where the HMRTN is an undirected graph.

(3) Regard the intersections as network nodes and the paths between neighboring nodes as network edges.

(4) Regard the distance between two nodes as the network edge weight.

According to the above analysis, hazardous materials road transportation complex network model based on the primal approach has the following properties: (1) network model comes from the real world, so the network structure is subjected to real network. (2) Nodes in network model have clear spatial location and coordinate. (3) The number of edges that network nodes can connect is influenced by road condition and the surrounding environment, which influences the distribution of node degrees.

2.2.2. The Service Network Model of HMRTN Based on Neighboring Nodes. Service network is a complex network consisting of a large number of OD pairs intersecting with each other, which reflects the relationships among different nodes and properties of HMRTN well. In this paper, a hazardous materials complex network model based on the neighboring nodes is constructed, and the following basic definitions and assumptions are made to the network topology.

(1) Regard the places of actual meaning like hazardous materials distribution center, demand center, and so forth as network nodes and distribution routes between neighboring nodes as the network edges.

(2) Regard the number of transportation vehicles passing the edge as network edge weight.

(3) Vehicle distribution go-return routes affect the network edge weight. For example, for a path from the distribution center to the demand center passing neighboring nodes $v_{m}$ and $v_{n}$, there is an edge $v_{m} \rightarrow$ $v_{n}$ between them; in the same way, when returning from the demand center to the distribution center, there is another edge $v_{n} \rightarrow v_{m}$ passing neighboring nodes $v_{m}$ and $v_{n}$.

(4) Do not consider the distribution volume of transportation vehicles, that is, no great difference in transport capacity of the transportation routes, and neighboring nodes have the same network edge weights.

(5) The tour-route distribution mode is used between the distribution center and demand center, and distribution center fully meets the demand of the transportation needs of demand center.
The properties of hazardous materials complex network model based on the neighboring nodes are as follows: (1) network nodes are a part of the physical network; they have accurate geographical location and spatial information; (2) network structure is subjected to physical network; (3) network node degree is the characterization of the service network, and the change of distribution task will directly affect the network degree distribution; (4) risk value, cost, and time need to be considered when choosing network distribution route, which has a direct influence on its complex network properties.

2.3. Methodology. Basic information for the study of HMRTN topological properties is network planning map and land-use map, but it cannot be directly used in calculation and analysis. Therefore, further calculation and processing are needed, and the specific steps are as follows.

Step 1. Complete the data processing using Arc-GIS software according to the city planning map and land-use map, and get the structure model of HMRTN.

Step 2. Construct topological structure of distribution network based on transportation network structure model according to hazardous materials distribution route data.

Step 3. Find the network topological adjacency matrix according to the distribution network topology data using the construction method of hazardous materials complex network model based on neighboring nodes.

Step 4. Calculate the network average node degree, average clustering coefficient, and degree distribution using topological adjacency matrix in the Matlab R2008a.

Step 5. Judge the complex network type and analyze the network topological properties.

\section{The Eigenvalues Calculation and Topological Properties Analysis of Complex Network of Hazardous Materials Road Transportation}

\subsection{The Physical Network of HMRTN}

3.1.1. The Basic Data of Physical Network of Dalian HMRTN. The Dalian network planning data in December 2013 is used in this paper, which contains 154 intersections and 238 paths. According to the construction method of hazardous materials road transportation physical network model based on the primal approach, Dalian hazardous materials road transportation physical complex network model is finally obtained by using Arc-GIS 10.0 to complete the process of spatial data, which is shown in Figure 2.

3.1.2. The Eigenvalues Calculation. Combined with Dalian hazardous materials road transportation physical complex network model, based on obtaining the network topological 
TABLE 1: Parameters of Dalian HMRTN.

\begin{tabular}{lcccccccc}
\hline Network eigenvalues & $m_{a}$ & $n_{a}$ & $\langle k\rangle$ & $L(\mathrm{~km})$ & $T(\mathrm{~km})$ & $C$ & $L_{R}(\mathrm{~km})$ & $C_{R}$ \\
\hline Calculation results & 154 & 238 & 3.0909 & 64.4485 & 193.3871 & 0.1714 & 4.1871 & 0.0216 \\
\hline
\end{tabular}

$m_{a}$ is the number of hazardous materials road transportation physical network's node, $n_{a}$ is the number of hazardous materials road transportation physical network's edge, $\langle k\rangle$ is the average node degree of anetwork, $L$ is the network characteristic path length, $T$ is the network diameter, $C$ is the network average clustering coefficient, $L_{R}$ is the characteristic path length of random network, and $C_{R}$ is network average clustering coefficient of random network.

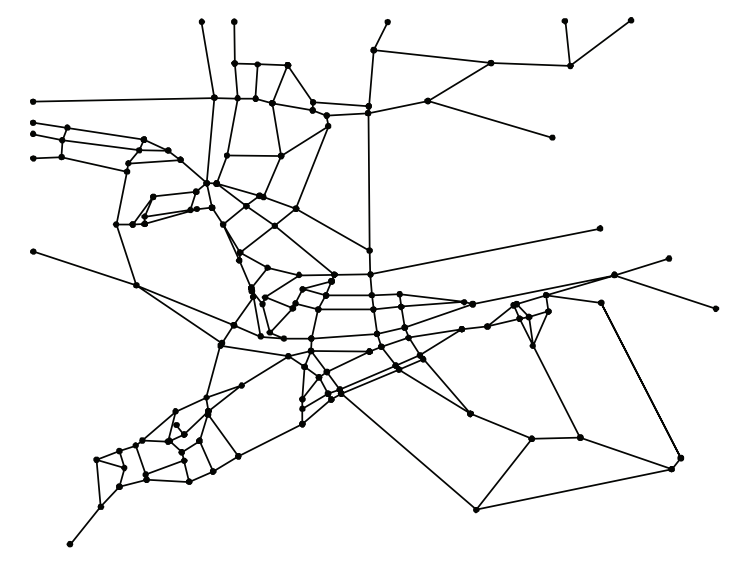

Figure 2: Physical network model of Dalian HMRTN based on primal approach.

adjacency matrix and distance adjacency matrix, the network eigenvalues such as the number of nodes, the number of edges, the network average node degree, characteristic path length, and network average clustering coefficient values, as well as the random network characteristic path length and network average clustering coefficient of the same scale, are calculated; the results are shown in Table 1.

The results in Table 1 show the following.

(1) There are 154 nodes and 238 edges in Dalian HMRTN, and the network average node degree is about 3.0909, between 3 4, which indicates that each intersection in the physical network is connected directly with an average of 3 paths. The average distance between any two nodes is $64.4485 \mathrm{~km}$, and the largest shortest distance between two nodes is $193.3871 \mathrm{~km}$.

(2) The physical network has a larger network average clustering coefficient, but it is much smaller than that of the urban road network [23].

(3) The characteristic path length $L$ and network average clustering coefficient $C$ of physical network are greater than those of random network of the same scale, which is consistent with the requirements $L \geq$ $L_{R}$ and $C \gg C_{R}$ [27]. Therefore, hazardous materials road transportation physical network topological structure presents small-world properties.

\subsubsection{The Topological Properties Analysis}

(1) The Nodes Degree Distribution. Analyzing the network node degree distribution is helpful for understanding spatial distribution of network nodes and the access rules of new
TABLE 2: The nodes degree statistics of physical network of Dalian HMRTN.

\begin{tabular}{lccccc}
\hline Node degree & 1 & 2 & 3 & 4 & 5 \\
\hline Node & 16 & 2 & 90 & 44 & 2 \\
Ratio/\% & 10.39 & 1.30 & 58.44 & 28.57 & 1.30 \\
\hline
\end{tabular}

nodes. Through the statistics of Dalian hazardous materials road transportation physical network model, the statistical results of network node degree are shown in Table 2. By calculating the network node degree and node degree cumulative distribution probability, and using Matlab Curve Fitting toolbox for data fitting, the diagram is shown in Figure 3.

In Table 2, nodes with node degree value below 3 in Dalian road transportation physical complex network account for $70.13 \%$ in the network, and nodes with node degree value above 5 only account for $1.30 \%$ in the network, which indicates that the network node degree distribution is not uniform, and the majority node degrees are generally small. The minimum value of node degree is 1 , which is induced by some nodes in the boundary of network. The maximum value of node degree is 5 , which shows that intersections connect with up to 5 paths in hazardous materials transportation network nodes.

In Figure 3, the network cumulative node degree distribution probability is subjected to exponential distribution, and the imitative effect is good. Known from [27], the connections among a new node and existing nodes in physical network are random, and network topological structure presents random network properties. This shows that the physical network is a tree network, and it is very easy to form isolated subgraphs after attack. And the low percolation point of tree network also means that the connectivity of physical network could be easily destroyed after attack.

(2) The Network Clustering Coefficient and Characteristic Path Length. The network average clustering coefficient and the characteristic path length are important indexes of network topological properties. In this paper, network topological structure properties of HMRTN are researched by observing the relationship among network characteristic path length, network average clustering coefficient, and network growth. The statistical results are shown in Figures 4 and 5.

In Figure 4, the linear fitting between network characteristic path lengths and the logarithm of nodes is good, and the fitting curve presents positive relationship, which shows that hazardous materials road transportation physical network presents property of small path length in the growth process.

In Figure 5, the ratio increases with the network node number increasing, and the fitting effect is very good. 


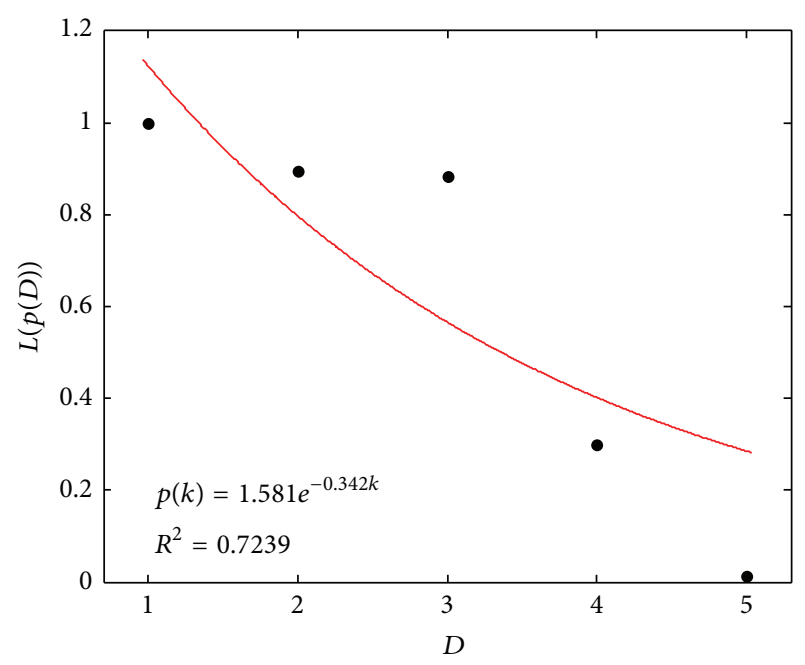

FIGURE 3: Probability distribution of cumulative node degree.

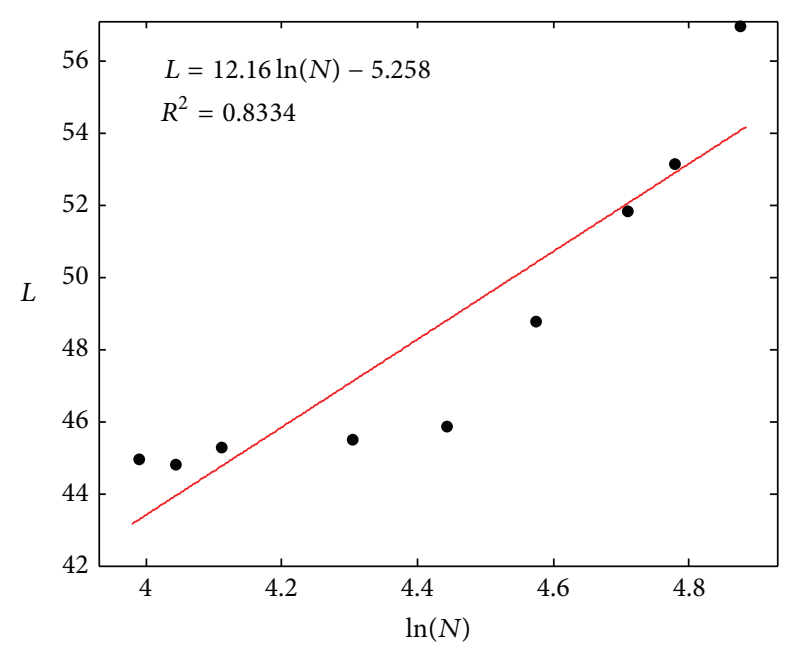

FIgURE 4: Curve of characteristic path length and $\ln (N)$.

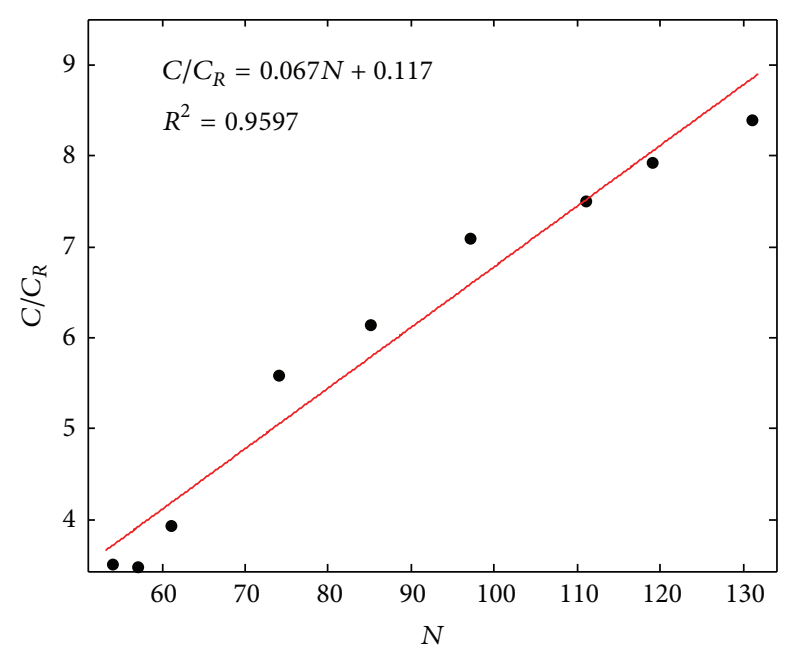

Figure 5: Curve of $C / C_{R}$ and network nodes.

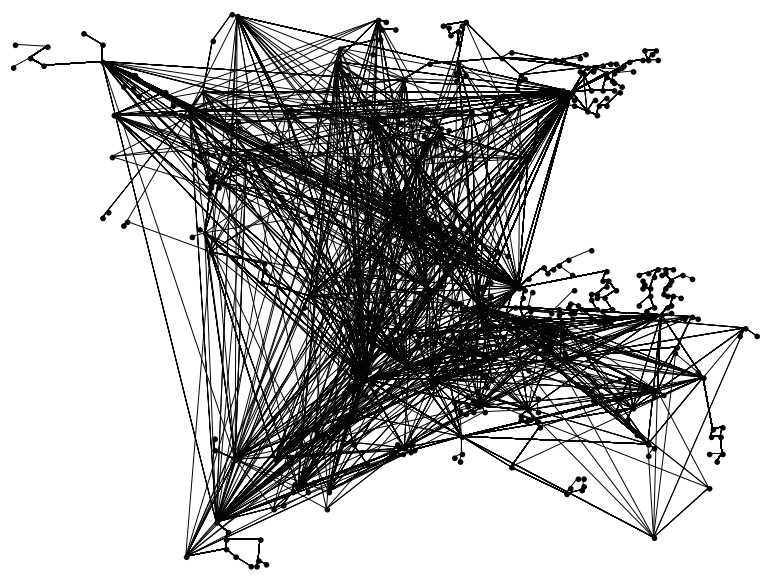

FIGURE 6: Service network model of Dalian HMRTN based on neighboring nodes.

It shows that the larger the scale of hazardous materials road transportation physical network is, the more obvious highly clustering properties of network topology are.

From the above results, Dalian hazardous materials road transportation physical network has small characteristic path length and large clustering coefficient, and it is a typical smallworld network. Small characteristic path length shows that the amount of information the physical network contains is not complex although its scale may be large, which makes it possible for people's cognition of physical network space. Large clustering coefficient shows that the physical network has good connectivity.

\subsection{The Service Network of HMRTN}

3.2.1. The Basic Data of Service Network of Dalian HMRTN. In all the impact factors of the planning for hazardous materials road transportation distribution route, safety is the most important one, which is distribution route of minimum risk. Therefore, hazardous materials road transportation service network route is subjected to not only physical network but also the network risk.

Matching with the operation data of Dalian hazardous materials road transportation physical network, Dalian hazardous materials distribution data in December 2013 is used in this paper. The distribution network includes 1318 paths and 569 network nodes. According to the construction method of hazardous materials road transportation service network model based on the neighboring nodes, Dalian hazardous materials road transportation service complex network model is finally obtained by using Arc-GIS 10.0, which is shown in Figure 6.

3.2.2. The Eigenvalues Calculation and Topological Properties Analysis. Known from Section 2.2.2, the service network constructed in this paper is a directed network, so the node degree is divided into out degree and in degree. Due to the fact that the round-trip routes of hazardous materials transportation vehicles are generally the same, there are no 
TABLE 3: The node degree statistics of service network of Dalian HMRTN.

\begin{tabular}{lcccccccccccc}
\hline Node degree & 1 & 2 & 3 & 4 & 5 & 6 & 7 & 8 & 9 & 10 & 11 & 12 \\
\hline Node & 180 & 175 & 70 & 30 & 11 & 6 & 6 & 5 & 13 & 3 & 5 & 3 \\
Ratio/\% & 31.63 & 30.76 & 12.30 & 5.27 & 1.93 & 1.05 & 1.05 & 0.88 & 2.28 & 0.53 & 0.88 & 0.53 \\
Node degree & 13 & 14 & 15 & 16 & 17 & 18 & 19 & 20 & 21 & 22 & 23 & 24 \\
Node & 2 & 4 & 5 & 3 & 8 & 3 & 5 & 6 & 4 & 2 & 3 & 3 \\
Ratio/\% & 0.35 & 0.70 & 0.88 & 0.53 & 1.41 & 0.53 & 0.88 & 1.05 & 0.70 & 0.35 & 0.53 & 0.53 \\
Node degree & 25 & 26 & 27 & 28 & 34 & 35 & 38 & 40 & 44 & 45 & 46 & 47 \\
Node & 2 & 2 & 1 & 1 & 1 & 1 & 1 & 1 & 1 & 1 & 1 \\
Ratio/\% & 0.35 & 0.35 & 0.18 & 0.18 & 0.18 & 0.18 & 0.18 & 0.18 & 0.18 & 0.18 & 0.18 & 0.18 \\
\hline
\end{tabular}

obvious differences between network node out degree and in degree in the service network. Based on this, network out degree will be regarded as the research object in this paper.

(1) The Node Degree and Node Degree Distribution. For hazardous materials road transportation service network based on the neighboring nodes, the node degree $D\left(v_{i}\right)$ of any node $v_{i}$ represents the number of such nodes as distribution center, demand center, and so forth that are directly connected with $v_{i}$. Therefore, it does not include edge weight. The bigger $D\left(v_{i}\right)$ is, the stronger the connectivity of this node is. And in practice, this node may be demand center or secondary distributor in great demand of category and quantity. Through the statistics of Dalian hazardous materials road transportation service network model, the statistical results of network node degree are shown in Table 3, and by using Matlab Curve Fitting toolbox for data fitting, the network node degree and degree distribution probability diagram are shown in Figure 7.

From the statistical results in Table 3, the maximum node degree of Dalian hazardous materials road transportation service complex network is 47 , and the average is about 4.63 , which shows that one node connects with an average of 5 nodes in the distribution process. The nodes with node degrees below 3 account for $74.69 \%$ in the network, and the nodes with node degrees above 30 account for only $1.41 \%$ in the total number of nodes, presenting uniform distribution.

In Figure 7, the node degree distribution $p(D)$ decreases with the change of node degree $D$ in power-law exponential forms, $p(D) \propto D^{-\beta}$, and $\beta \approx 1$ 156. Known from [27], hazardous materials road transportation service network based on the neighboring nodes is subjected to powerlaw distribution, representing the properties of scale-free network.

For further study on complex network properties of the network nodes, the node degree and its cumulative distribution are dealt with by double logarithmic processing, and the fitting results are shown in Figure 8.

Through double logarithmic processing of node degree and its cumulative distribution probability and linear regression fitting of the results, the equation can be obtained as $\log (p(D))=-1.481 \log (D)+5.651$, where the coefficient $R^{2}=$ 0.8639 . The statistical results show that hazardous materials road transportation service network is subjected to powerlaw distribution.

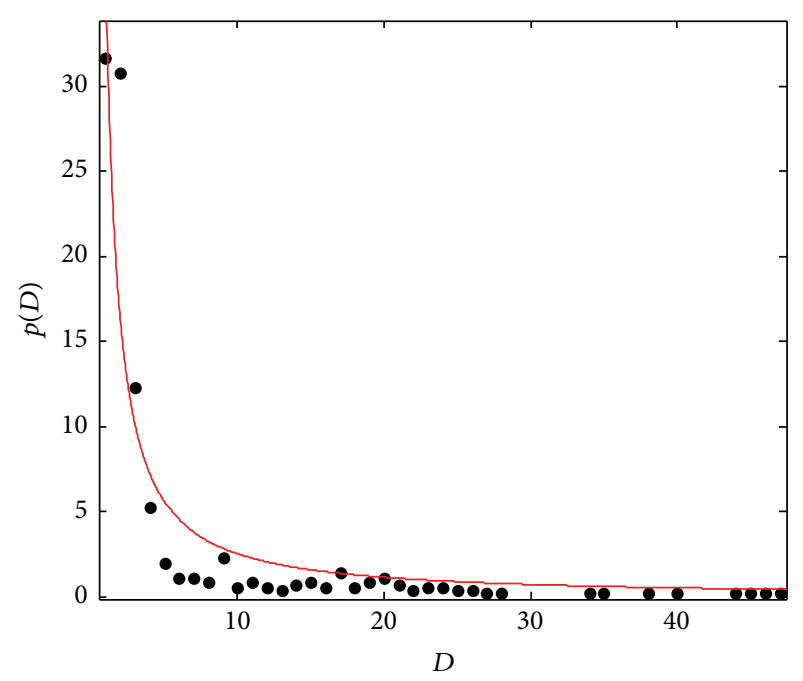

FIGURE 7: The relationship between nodes degree and node degree distribution of service network of Dalian HMRTN.

According to the above analysis, in hazardous materials road transportation service network based on the neighboring nodes, the relationship between node degree and degree distribution, and the double logarithmic relationship between node degree and cumulative node degree distribution probability are both subjected to power-law distribution and present scale-free network properties.

(2) The Node Strength. Analysis on network node strength distribution can help to understand not only the connected relationship among network nodes but also the network traffic density and the connectivity among network nodes. Service network is a network of weighted edges, and the weight represents the efficiency of paths between neighboring nodes. Referring to [28], hazardous materials road transportation service network node strength can be defined as follows in this paper:

$$
D^{\prime}\left(v_{i}\right)=\sum_{v_{j} \in V_{v_{i}}^{\prime}} w_{v_{i} v_{j}},
$$

where $D^{\prime}\left(v_{i}\right)$ is the node strength of node $v_{i}, v_{j} \in V_{v_{i}}^{\prime}$ is node $v_{j}$ directly connected with node $v_{i}$, and $w_{v_{i} v_{j}}=0$ or 1 , where 
TABLE 4: The node strength statistics of service network of Dalian HMRTN.

\begin{tabular}{lccccccccccc}
\hline Node strength & 1 & 2 & 3 & 4 & 5 & $\ldots$ & 16 & 17 & 18 & 19 \\
\hline Node & 50 & 18 & 16 & 11 & 9 & $\ldots$ & 6 & 4 & 3 & 2 \\
Ratio/\% & 12.92 & 4.65 & 4.13 & 2.84 & 2.33 & $\ldots$ & 1.55 & 1.03 & 0.78 & 0.52 & 1.55 \\
Node strength & 68 & 69 & 70 & 71 & 72 & $\ldots$ & 155 & 194 & 195 & 223 & 289 \\
Node & 6 & 4 & 1 & 2 & 2 & $\ldots$ & 1 & 1 & 1 & 1 & 1 \\
Ratio/\% & 1.55 & 1.03 & 0.26 & 0.52 & 0.52 & $\ldots$ & 0.26 & 0.26 & 0.26 & 0.26 & 0.26 \\
\hline
\end{tabular}

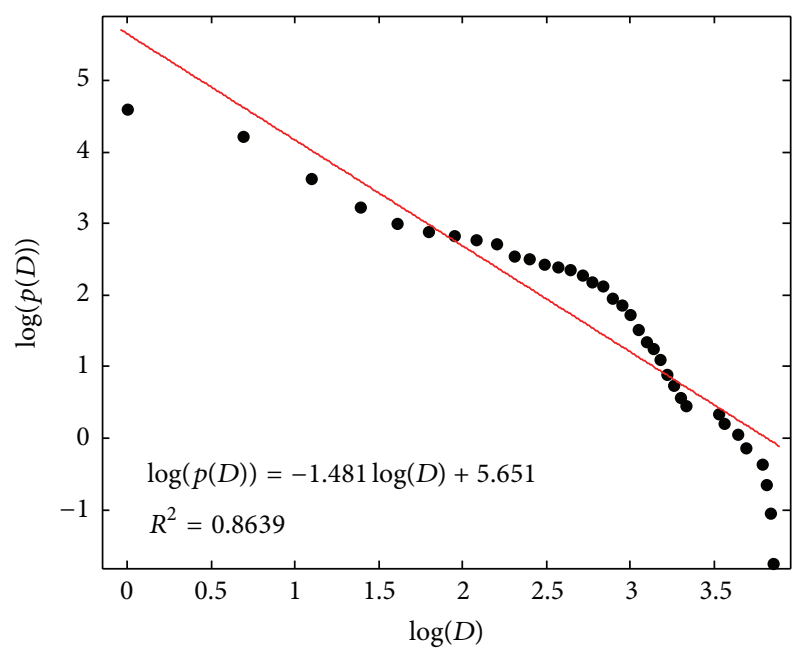

FIGURE 8: The relationship between nodes degree and cumulative node degree.

an edge exists between nodes $v_{i} \rightarrow v_{j}, w_{v_{i} v_{j}}=1$, and otherwise $w_{v_{i} v_{j}}=0$.

According to (1), the network node strength distribution can be defined as follows:

$$
\prod\left(D^{\prime}\left(v_{i}\right)\right)=\frac{D^{\prime}\left(v_{i}\right)}{N},
$$

where $\prod\left(v_{i}\right)$ is the distribution probability of node strength equal to $D^{\prime}\left(v_{i}\right)$ and $N$ is the sum of network node strengths.

Through the statistics of Dalian hazardous materials road transportation service network, the statistical results of the network node strengths are shown in Table 4.

Through the analysis of the data in Table 4 , the average node strength $\left\langle D^{\prime}\right\rangle$ of the service network is 29.53 , and the maximum node strength is 289 . This shows that about 30 distribution routes go through each node. With an average of 1 node connected with 5 nodes; therefore, the average strength of two neighboring nodes is 6, which shows that an average of 6 distribution routes go through two neighboring nodes.

In Table 4 , the top $20 \%$ nodes contribute $52.20 \%$ of accumulative node strength and the top $10 \%$ of cumulative node strength has reached $38.50 \%$ in the 569 nodes of Dalian hazardous materials road transportation service network. This shows that the normal operation of the whole service network is maintained by only a few nodes. Otherwise, the cumulative node strength of nodes with node strength below 5 is up to $26.87 \%$, which indicates that most of the nodes

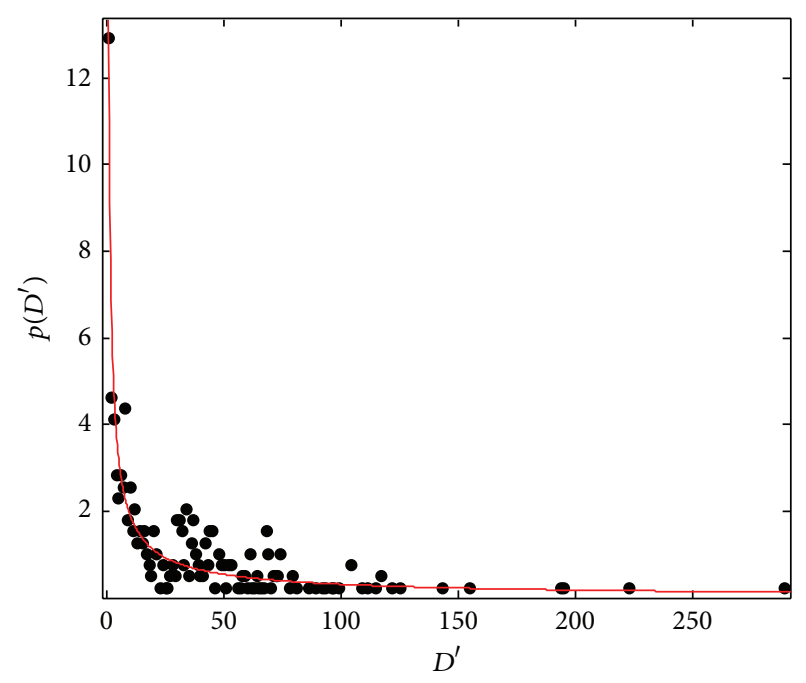

Figure 9: The relationship between nodes strength and nodes strength distribution of service network of Dalian HMRTN.

in the service network are normal nodes. The distribution task through these nodes is relatively small, but their presence increases the coverage and density of the service network.

(3) The Node Strength Distribution. In order to research the complex network properties of the of network node strength, the statistics of Dalian hazardous materials road transportation service network node strengths are carried out in this paper, and the nodes strength and their strength distribution probability are shown in Figure 9.

In Figure 9, the node strength distribution $p\left(D^{\prime}\right)$ decreases with the change of node strength $D^{\prime}$ in power-law exponential forms, $p\left(D^{\prime}\right) \propto D^{\prime-\beta}$, and $\beta \approx 0.7751$. Known from [27], hazardous materials road transportation service network is generally subjected to power-law distributions and represents the properties of scale-free network.

For further study on internal dynamics properties of the network nodes, the relationship between service network node strength with intensity sequence number and cumulative node strength will be examined. Figure 10 shows the nodes strength changing with its descending order ordinal, and Figure 11 shows the node strength changing with the cumulative node strength increasing.

In Figure 10, the node strength and its descending order ordinal are dealt with by double logarithmic processing. Using Matlab Curve Fitting toolbox for linear regression fitting, the equation can be obtained as 


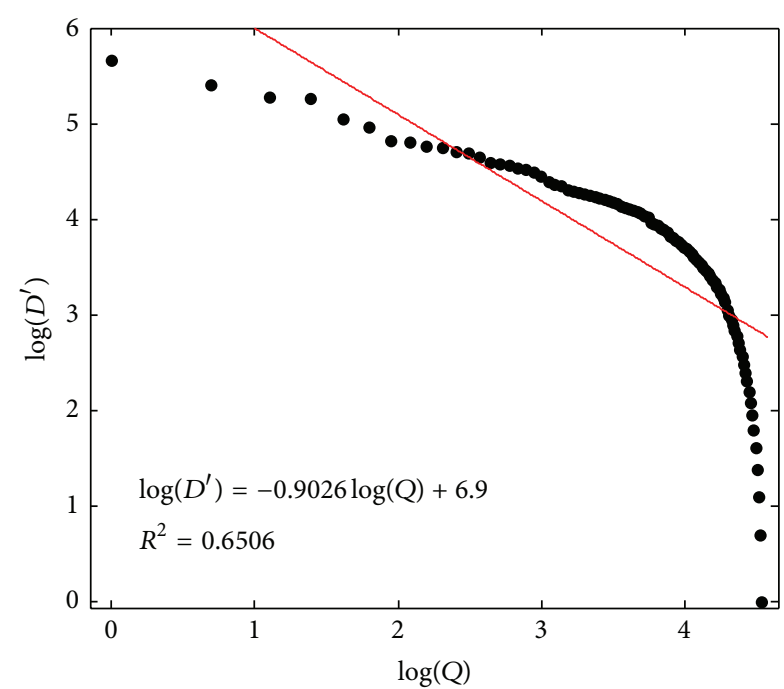

FIGURE 10: The relationship between nodes strength and its descending order ordinal of service network of Dalian HMRTN.

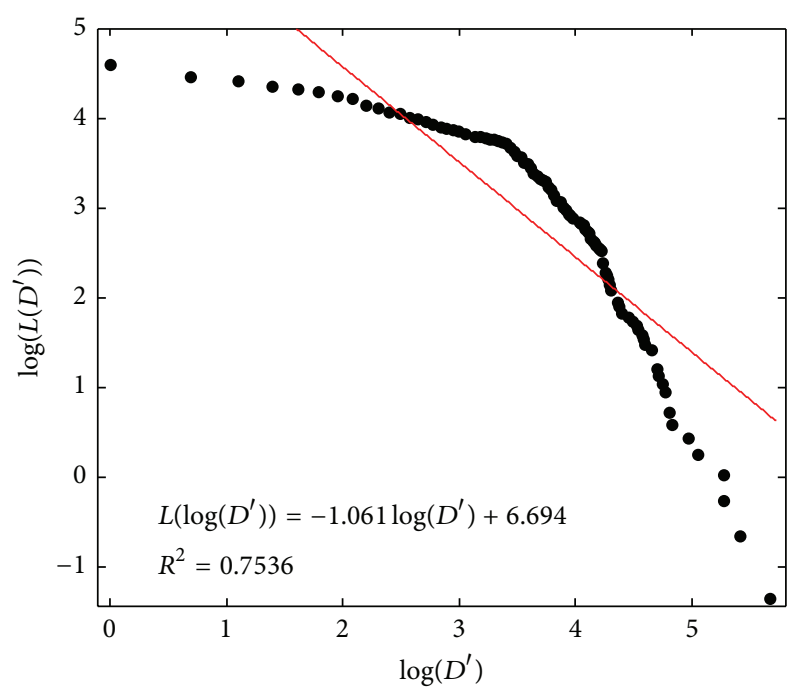

FIGURE 11: The relationship between nodes strength and cumulative nodes strength of service network of Dalian HMRTN.

$\log \left(D^{\prime}\right)=-0.9026 \log (Q)+6.9$ and the coefficient as $R^{2}=0.6506$. The above statistical results show that the node strength and its descending order ordinal are subjected to power-law distribution on the whole.

In Figure 11, the nodes strength and cumulative nodes strength are dealt with by double logarithmic processing. Using Matlab Curve Fitting toolbox for linear regression fitting, the equation can be obtained as $L\left(\log \left(D^{\prime}\right)\right)=$ $-1.061 \log \left(D^{\prime}\right)+6.694$ and the coefficient as $R^{2}=0.7536$. The above statistical results show that the nodes strength and cumulative nodes strength are also subjected to power-law distribution on the whole.

According to the above analysis, in the hazardous materials road transportation service network based on neighboring nodes, node strength and its distribution probability, node strength and its descending order ordinal, and node strength and cumulative node strength distribution probability are all subjected to power-law distribution and represent properties of scale-free network. It presents that the production, circulation, and distribution of hazardous materials are completed by only a few nodes in the service network, which reflects the internal dynamic properties and essence of the service network.

\section{Conclusions}

To research the topological properties of HMRTN, this paper proposes two different ways to construct the cyberspace of HMRTN and constructs its complex network models, respectively. The Dalian HMRTN is regarded as a researching case. Using the complex network theory and statistical analysis, the main conclusions are as follows.

(1) By analyzing hazardous materials road transportation physical network, its node degree distribution in Dalian is very uneven. The cumulative node degree of physical network is subjected to exponential distribution, showing the network properties of random network; network characteristic path length and clustering coefficient change with the network scale, showing small-world network properties. These results reflect the complexity and the internal dynamic properties of hazardous materials road transportation physical network.

(2) The random network properties of physical network show that each node of physical connection in Dalian is random connection. It could be considered that, in HMRTN planning, urban long and wide main roads are typically preferred, and they should be away from populated areas with high road network density, which also leads to low connectivity between physical network nodes. Therefore, we must strengthen the construction of hazardous materials road transportation infrastructure and improve the network average node degree to ensure the connection and reliability of hazardous materials road transportation.

(3) The network average clustering coefficient represents the structure properties of the physical network to some extent; the greater its numerical value is, the better the connectivity of the network would be. Although the physical network presents small-world properties, the network average clustering coefficient is much smaller compared to other transportation networks. When the network paralysis occurs in some key nodes and edges, it would be very easy to make the nodes isolated. Therefore, improving the network average clustering coefficient of a physical network is an effective way to optimize the topological properties of hazardous materials road transportation physical network. 
(4) By analyzing hazardous materials road transportation service network, its node degree and node strength distribution in Dalian are very uneven. The relationship between node degree and cumulative node degree of service network is subjected to power-law distribution, showing the network properties of scalefree network. The relationship among nodes strength, its descending order ordinal, and cumulative nodes strength are all subjected to power-law distribution, showing the network properties of scale-free network. These results reflect the complexity and the internal dynamic properties of hazardous materials road transportation service network. The results can also be used as an example of scale-free network growth model.

(5) The scale-free network properties presented by service network node strength show that hazardous materials in the whole process from production to consumption are always completed by only a few companies. And those companies' transport once blocked will cause the paralysis to the whole service network transportation efficiency. Therefore, we should try our best to reduce the pressure of the key companies, which can enhance the survivability of the whole network under the unexpected events.

(6) Service network node degree mainly emphasizes the connectivity between network nodes, while node strength presents distribution load. Therefore, there is difference between service network node degree and node strength ranking, and this difference reflects the contradiction between the planned network and distribution pressure. So service network node degree and node strength can be used as reference to the optimization of distribution.

This paper is only a preliminary study on hazardous materials road transportation complex network topological properties. HMRTN is a typical open complex network with road system and distribution system, which are mutually influenced and interdependent, and it is a super network including physical network and service network. Therefore, the coupling relationship between physical network and service network of hazardous material road transportation and network planning optimization will be the work of next step.

\section{Conflict of Interests}

The authors declare that there is no conflict of interests regarding the publication of this paper.

\section{Acknowledgment}

The authors would like to appreciate the financial support from the National Natural Science Foundation of China (no. 71173177).

\section{References}

[1] M. Rubinov and O. Sporns, "Complex network measures of brain connectivity: Uses and interpretations," NeuroImage, vol. 52, no. 3, pp. 1059-1069, 2010.

[2] R. Albert, H. Jeong, and A.-L. Barabási, "Error and attack tolerance of complex networks," Nature, vol. 406, no. 6794, pp. 378-382, 2000.

[3] J. A. Dunne, R. J. Williams, and N. D. Martinez, "Network structure and biodiversity loss in food webs: robustness increases with connectance," Ecology Letters, vol. 5, no. 4, pp. 558-567, 2002.

[4] L. A. N. Amaral, A. Scala, M. Barthélémy, and H. E. Stanley, "Classes of small-world networks," Proceedings of the National Academy of Sciences of the United States of America, vol. 97, no. 21, pp. 11149-11152, 2000.

[5] S. Redner, "How popular is your paper? An empirical study of the citation distribution," The European Physical Journal BCondensed Matter and Complex Systems, vol. 4, no. 2, pp. 131134, 1998.

[6] S. Milgram, "The small world problem," Psychology Today, vol. 1, no. 1, pp. 61-67, 1967.

[7] D. J. Watts and S. H. Strogatz, "Collective dynamics of "smallworld” networks," Nature, vol. 393, no. 6684, pp. 440-442, 1998.

[8] A.-L. Barabási and R. Albert, "Emergence of scaling in random networks," Science, vol. 286, no. 5439, pp. 509-512, 1999.

[9] M. Metcalfe, "Knowledge sharing, complex environments and small-worlds," Human Systems Management, vol. 24, no. 3, pp. 185-195, 2005.

[10] V. Latora and M. Marchiori, "Is the Boston subway a smallworld network?," Physica A: Statistical Mechanics and Its Applications, vol. 314, no. 1-4, pp. 109-113, 2002.

[11] K. A. Seaton and L. M. Hackett, "Stations, trains and smallworld networks," Physica A: Statistical Mechanics and its Applications, vol. 339, no. 3-4, pp. 635-644, 2004.

[12] P. Sen, S. Dasgupta, A. Chatterjee, P. A. Sreeram, G. Mukherjee, and S. S. Manna, "Small-world properties of the Indian railway network," Physical Review E: Statistical, Nonlinear, and Soft Matter Physics, vol. 67, no. 3, Article ID 036106, 2003.

[13] W. Li and X. Cai, "Statistical analysis of airport network of China," Physical Review E, vol. 69, no. 4, Article ID 046106, 2004.

[14] M. Kurant and P. Thiran, "Extraction and analysis of traffic and topologies of transportation networks," Physical Review E, vol. 74, no. 3, Article ID 036114, 2006.

[15] R. Guimerà and L. A. N. Amaral, "Modeling the world-wide airport network," European Physical Journal B, vol. 38, no. 2, pp. 381-385, 2004.

[16] R. Guimerà, S. Mossa, A. Turtschi, and L. N. Amaral, "The worldwide air transportation network: anomalous centrality, community structure, and cities' global roles," Proceedings of the National Academy of Sciences of the United States of America, vol. 102, no. 22, pp. 7794-7799, 2005.

[17] L.-P. Chi, R. Wang, H. Su et al., "Structural properties of US flight network," Chinese Physics Letters, vol. 20, no. 8, pp. 13931396, 2003.

[18] G. Bagler, "Analysis of the airport network of India as a complex weighted network," Physica A: Statistical Mechanics and Its Applications, vol. 387, no. 12, pp. 2972-2980, 2008.

[19] M. Guida and F. Maria, "Topology of the Italian airport network: a scale-free small-world network with a fractal structure?" Chaos, Solitons \& Fractals, vol. 31, no. 3, pp. 527-536, 2007. 
[20] W. Li and X. Cai, "Statistical analysis of airport network of China," Physical Review E-Statistical, Nonlinear, and Soft Matter Physics, vol. 69, no. 4, Article ID 046106, 2004.

[21] B. Jiang and C. Claramunt, "Topological analysis of urban street networks," Environment and Planning B: Planning and Design, vol. 31, no. 1, pp. 151-162, 2004.

[22] P. Holme, B. J. Kim, C. N. Yoon, and S. K. Han, "Attack vulnerability of complex networks," Physical Review E: Statistical, Nonlinear, and Soft Matter Physics, vol. 65, no. 5, Article ID 056109, 2002.

[23] A. W. Krings and A. Azadmanesh, "A graph based model for survivability applications," European Journal of Operational Research, vol. 164, no. 3, pp. 680-689, 2005.

[24] J. J. Wu, H. J. Sun, and Z. Y. Gao, "Cascading failures on weighted urban traffic equilibrium networks," Physica A: Statistical Mechanics and Its Applications, vol. 386, no. 1, pp. 407-413, 2007.

[25] Economy Statistics for Chemical Engineering in China, http://wms.mofcom.gov.cn/article/ztxx/nyhg/hyyxqk /201307/ 20130700190421.shtml.

[26] S. Porta, P. Crucitti, and V. Latora, "The network analysis of urban streets: A dual approach," Physica A: Statistical Mechanics and Its Applications, vol. 369, no. 2, pp. 853-866, 2006.

[27] A.-L. Barabási, R. Albert, and H. Jeong, "Mean-field theory for scale-free random networks," Physica A: Statistical Mechanics and Its Applications, vol. 272, no. 1, pp. 173-187, 1999.

[28] S. H. Yook, H. Jeong, A.-L. Barabási, and Y. Tu, "Weighted evolving networks," Physical Review Letters, vol. 86, no. 25, pp. 5835-5838, 2001. 


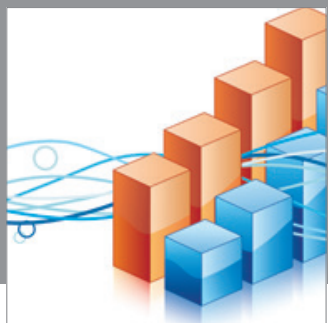

Advances in

Operations Research

mansans

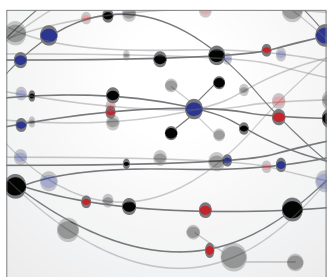

The Scientific World Journal
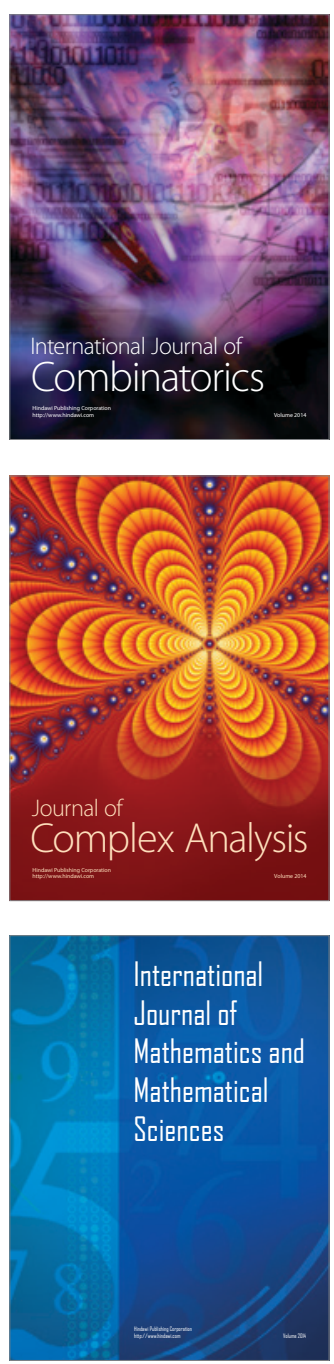
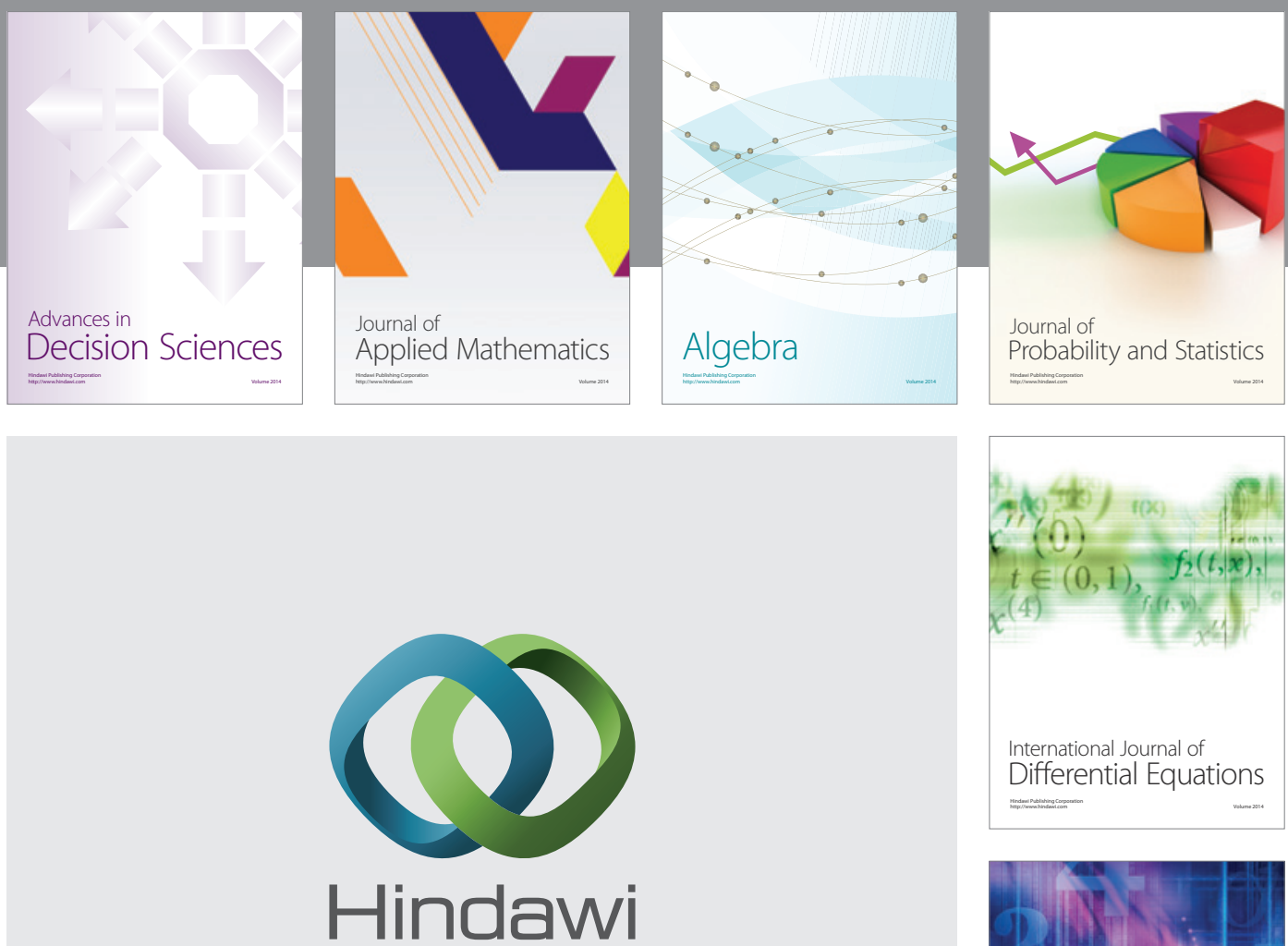

Submit your manuscripts at http://www.hindawi.com
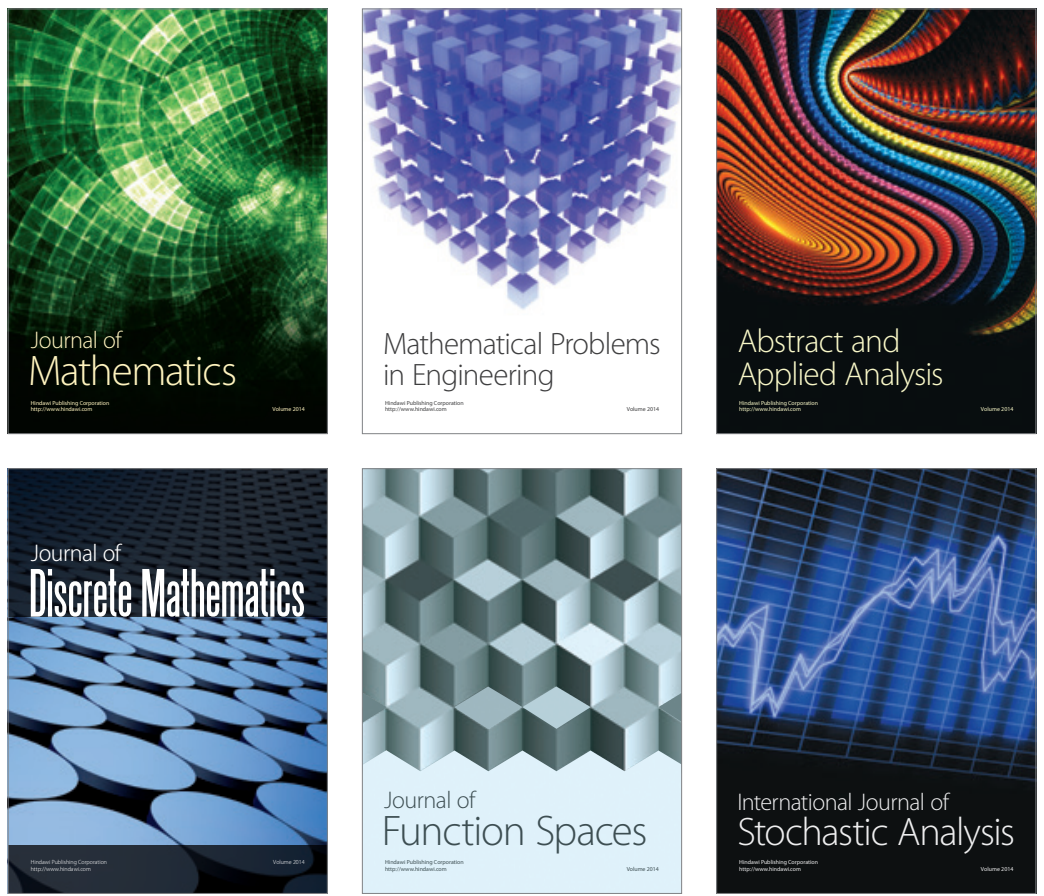

Journal of

Function Spaces

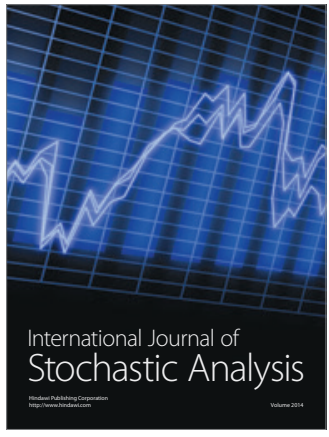

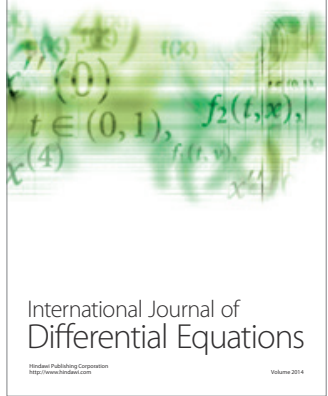
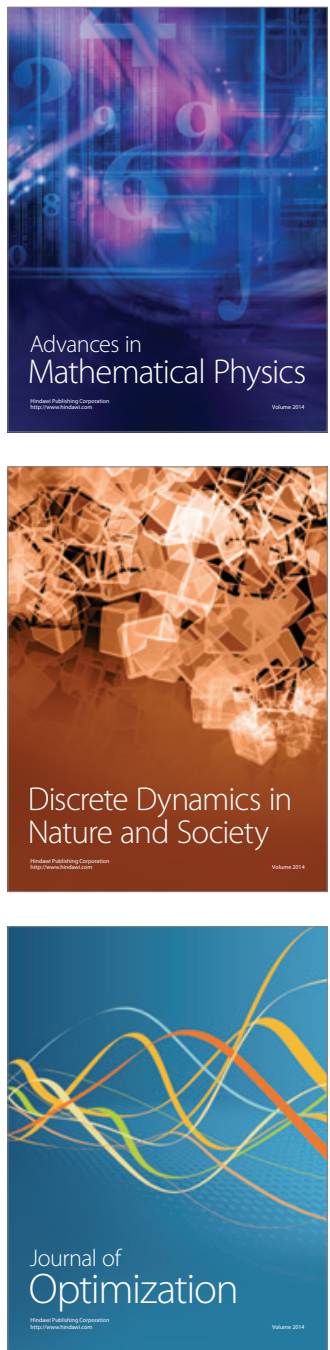